\title{
Balkanologie
}

Balkanologie Revue d'études pluridisciplinaires

Vol. II, nº 1 | 1998

Volume II Numéro 1

\section{Botchev (Stéphane), Béléné. Souvenirs du goulag} bulgare

Montricher : Ed. Noir sur Blanc, 1998, 278 p.

\section{Bernard Lory}

\section{(2) OpenEdition}

\section{Journals}

Édition électronique

URL : http://journals.openedition.org/balkanologie/249

DOI : 10.4000/balkanologie.249

ISSN : 1965-0582

\section{Éditeur}

Association française d'études sur les Balkans (Afebalk)

Édition imprimée

Date de publication : 1 juillet 1998

ISSN : 1279-7952

\section{Référence électronique}

Bernard Lory, «Botchev (Stéphane), Béléné. Souvenirs du goulag bulgare », Balkanologie [En ligne], Vol. II, $n^{\circ} 1$ | 1998, mis en ligne le 02 juin 2008, consulté le 17 décembre 2020. URL : http://

journals.openedition.org/balkanologie/249; DOI : https://doi.org/10.4000/balkanologie.249

Ce document a été généré automatiquement le 17 décembre 2020.

(c) Tous droits réservés 


\section{Botchev (Stéphane), Béléné. Souvenirs du goulag bulgare}

Montricher : Ed. Noir sur Blanc, 1998, 278 p.

Bernard Lory

\section{RÉFÉRENCE}

Botchev (Stéphane), Béléné. Souvenirs du goulag bulgare, Montricher : Ed. Noir sur Blanc, $1998,278 \mathrm{p}$.

1 Les témoignages sur les camps communistes ne manquent pas. Le public francophone a pu en lire concernant la Roumanie ${ }^{1}$, la Yougoslavie ${ }^{2}$ et la Bulgarie ${ }^{3}$. Il n'y a donc pas de révélation historique extraordinaire à attendre de l'ouvrage de Stefan Bočev, qui s'inscrit dans un genre à la fois documentaire et littéraire, dont on peut faire remonter la filiation à Dostoïevski.

2 Le souci documentaire n'est pas au cœur de la démarche de Bočev. Il écrit ses mémoires en 1984, plus de trente ans après les faits, et ne dispose plus des notes qu'il avait prises "à chaud" dans les camps. Ces notes, observe-t-il d'ailleurs, étaient souvent accaparées par des préoccupations climatiques ou de travail, d'une importance primordiale dans l'immédiat, mais sans intérêt pour le mémorialiste ${ }^{4}$.

3 Simultanément, Bočev n'a pas de prétention littéraire. Ses mémoires, rédigées au fil de la plume, comprennent de nombreuses digressions. Il abandonne les détenus de Belene au moment le plus dramatique d'une crue du Danube, pour entraîner le lecteur dans une promenade nostalgique à travers les rues de Sofia (pp. 175-190). Malgré ces imperfections au niveau de la composition, le récit est attachant et évocateur.

4 On reprochera cependant à Stefan Bočev de ne pas se présenter de façon directe. Des éléments autobiographiques éparpillés au cours du récit permettent toutefois de reconstituer son profil. Issu d'une famille riche, il a vécu longtemps à l'étranger, a côtoyé les cercles du pouvoir de près (il a été secrétaire administratif du Premier 
ministre Kjoseivanov), a été employé au ministère des Affaires étrangères et a sympathisé tardivement avec les agrariens de Nikola Petkov.

5 L'édition aurait été bien inspirée de rassembler cette information éparse dans une petite introduction, qui aurait permis au lecteur de cerner d'emblée le contexte social et politique de ce témoignage. De même, une chronologie sommaire de la vie politique bulgare entre 1920 et 1950 aurait-elle été bien utile au public francophone, car Bočev est souvent allusif. Les quelques notes en bas de page proposées par l'édition sont totalement insuffisantes.

Le lecteur saura gré à Stefan Bočev de lui avoir épargné les scènes de brutalité et de sadisme qui rendent certains ouvrages insoutenables (ceux de Ierunca et Popović en particulier). La bastonnade revient fréquemment, les exécutions sommaires aussi, mais le filtre de la mémoire a curieusement retenu, non les souffrances, mais les périodes d'euphorie physique que peuvent susciter la sous-alimentation et le travail exténuant. Bočev s'attarde avec un plaisir évident à évoquer ses travaux de bûcheronnage sur un îlot désert du Danube ou son activité de gardien de troupeau. Le soleil, l'eau, les animaux sauvages, la splendeur des crépuscules sont décrits avec une passion vibrante, qui finit par troubler le lecteur : les camps bulgares n'auraient-ils été qu'une variante brutale à l'extrême des camps scouts? Cette impression est bien sûr fallacieuse et déplacée. Elle provient de l'attitude résolument positive qu'adopte l'auteur envers son propre vécu. Il affirme même avoir "choisi" son destin, puisqu'en 1945 il aurait pu rester en Suisse. Ce ton distancié et apaisé donne tout son prix à ce témoignage : pas d'indignation, ni d'apitoiement, pas de froideur factuelle visant une impossible objectivité non plus.

7 Les souvenirs de Stefan Bočev nous apportent en outre une masse d'informations un peu diffuse sur la société bulgare de l'époque : la place qu'y occupaient les protestants ou les anarchistes, les pratiques démagogiques des politiciens agrariens et leur aveuglement international, etc. Certaines fulgurations nous font toucher le plus profond des représentations collectives, telle la comparaison de l'entrevue entre les détenus et leur famille avec un banquet funéraire (p. 258). Le lecteur est reconnaissant d'une vision du monde qui n'oppose pas mécaniquement bourreaux et victimes, mais qui recherche, envers et contre tout, une unité. Cette unité, Bočev veut la trouver dans une sorte de communion nationale. Profondément épris de l'idée du peuple bulgare (qu'une sorte d'épiphanie lui montre incarné dans une paysanne traversant les maïs, $p$. 153), il n'en exclut pas les miliciens.

8 Il voudrait croire que ces camps, où des gardiens bulgares broient des destins bulgares sur le sol bulgare, sont en fait des produits d'importation :

Écrire sur les camps n'est pas pour moi une fin en soi. Si j'écris sur les camps, c'est parce qu'ils font partie de la réalité bulgare, et que nous avons besoin de comprendre cette réalité. Il faut bien avouer, soit dit en passant, que ceux que je connus n'avaient de bulgare que la construction. Le modèle, lui, était bien soviétique. La rumeur courait même que des experts soviétiques assistaient, sans dire un mot, aux interrogatoires. Cela ne me paraît pas invraisemblable, car le "grand frère" pouvait difficilement nous laisser sans surveillance, mais je ne suis pas en mesure de le confirmer et je ne remarquai jamais rien de tel (p. 160).

9 La contradiction du propos est révélatrice : malgré toute son honnêteté intellectuelle, Bočev s'arrête sur le seuil d'une vérité qu'il ne peut se résoudre à formuler, à savoir que la violence communiste en Bulgarie est de nature endogène. Qu'il serait réconfortant d'avoir rencontré un instructeur soviétique expert ès-goulag, donnant des ordres et des 
consignes! Mais non! Tous les miliciens sont de pauvres Bulgares, maladroits et balourds, qui, visiblement, improvisent au jour le jour un monde concentrationnaire.

Le terme de goulag est utilisé de façon abusive pour désigner les prisons et les camps du régime communiste dans un pays comme la Bulgarie. On se souvient de ces affiches de propagande anti-communistes qui ont fleuri en 1990, où le territoire bulgare était constellé de crânes. Elles voulaient suggérer que le pays avait connu ce véritable monde parallèle, cet "archipel du goulag" qui est une caractéristique soviétique. Réduite aux dimensions de la petite Bulgarie, cette vision est ridicule. Les documents du ministère de l'Intérieur publiés depuis 1989 ont révélé que, de 1944 à 1962, 23531 personnes auraient été détenues en camps (21 442 hommes et 2089 femmes), dont 14647 pour des raisons politiques, 6190 pour des crimes de droit commun, 2694 pour des motifs inconnus ${ }^{5}$.

11 Ceux qui souhaitent se convaincre que, dans les Balkans, la cruauté concentrationnaire n'est pas un produit d'importation soviétique auront tout intérêt à lire les souvenirs du communiste grec Chronis Missios ${ }^{6}$. La défense de ce que l'on appelait à l'époque "le monde libre" a provoqué des “dérapages” d'un sadisme terrifiant. À ce propos, il n'est peut-être pas inutile de rappeler que la violence institutionnalisée a fait son apparition en Bulgarie en 1923-1926 sous les auspices d'un juriste et professeur de sciences politiques, Aleksandăr Cankov?

Mais ces réflexions nous éloignent du témoignage de Stefan Bočev. Ce n'est pas le problème de l'origine du mal qui le préoccupe en premier lieu, mais, de façon bien plus modeste, celui de recoller les fragments d'une existence que l'Histoire a brisée. Et son récit, sincère et émouvant, nous montre que cela n'est pas impossible.

\section{NOTES}

1. Wurmbrand (Richard), Mes prisons avec Dieu, Tournai, 1970 ; Orlea (Dana), Les années volées. Dans le goulag roumain à 16 ans, Paris, 1992 ; Ierunca (Virgil), Piteşti, laboratoire concentrationnaire (1949-1952), Paris, 1996.

2. Popovic (Miroslav), Les vauriens de Tito, Paris, 1988.

3. Stantchev (Michail), Sous le joug du libérateur, Moudon, 1965 ; Todorov (Tsvetan), éd., Au nom du peuple. Témoignages sur les camps communistes, La Tour d'Aignes, 1992.

4. Nous relevons par ailleurs quelques erreurs factologiques vénielles dans son texte:

- un poème de Karavelov est attribué à Slavejkov (p. 155),

- Iv. Ev. Gešov n'était pas « un jeune paysan de Yablanitza », mais un riche bourgeois de Plovdiv (p. 187),

- Čamkorija (Borovec) est au sud-est de Sofia (p. 124).

5. Enciklopedija Bălgarija, t. VII, Sofia, 1996, supplément (article «Trudovovăzpitatelni obštežitija »).

6. Missios (Chronis), Toi au moins, tu es mort avant, La Tour d'Aignes, 1991.

7. Barbusse (Henri), Les bourreaux, Paris, 1926. 